\title{
THE RICH MID-INFRARED ENVIRONMENTS OF TWO HIGHLY OBSCURED X-RAY BINARIES: SPITZER OBSERVATIONS OF IGR J16318-4848 AND GX 301-2
}

\author{
Dae-Sik Moon, ${ }^{1}$ David L. Kaplan, ${ }^{2}$ William T. Reach, ${ }^{3}$ Fiona A. Harrison, ${ }^{4}$ Jeong-Eun Lee, ${ }^{5}$ and Peter G. Martin ${ }^{6}$ \\ Received 2007 July 9; accepted 2007 October 19; published 2007 November 7
}

\begin{abstract}
We present the results of Spitzer spectroscopic observations of two highly obscured massive X-ray binaries: IGR J16318-4848 and GX 301-2. Our observations reveal for the first time the extremely rich mid-infrared environments of this type of source, including multiple continuum emission components (a hot component with $T>700 \mathrm{~K}$ and a warm component with $T \sim 180 \mathrm{~K}$ ) with apparent silicate absorption features, numerous $\mathrm{H}$ I recombination lines, many forbidden lines of low ionization potential, and pure rotational $\mathrm{H}_{2}$ lines. This indicates that both sources have hot and warm circumstellar dust, ionized stellar winds, extended low-density ionized regions, and photodissociated regions. It appears difficult to attribute the total optical extinction of both sources to the hot and warm dust, which suggests that there could be an otherwise observable colder dust component responsible for most of the optical extinction and silicate absorption features. The observed mid-infrared spectra are similar to those from luminous blue variables, indicating that the highly obscured massive X-ray binaries may represent a previously unknown evolutionary phase of X-ray binaries with early-type optical companions. Our results highlight the importance and utility of mid-infrared spectroscopy for investigating highly obscured X-ray binaries.
\end{abstract}

Subject headings: circumstellar matter — infrared: stars — stars: emission-line, Be $-\mathrm{X}$-rays: binaries X-rays: individual (GX 301-2, IGR J16318-4848)

\section{INTRODUCTION}

Recently, a large number of highly obscured (e.g., $N_{\mathrm{H}} \geq 10^{23}$ $\mathrm{cm}^{-2}$ ) massive X-ray binaries have been discovered by the $I N$ TEGRAL hard X-ray ( $\geq 15 \mathrm{keV}$ ) satellite (Winkler et al. 2003). The prototypical case is IGR J16318-4848, which shows variable high obscuration in the X-ray, sometimes reaching $N_{\mathrm{H}} \simeq 2 \times$ $10^{24}$ (Courvoisier et al. 2003; Walter et al. 2003). Its bright optical and near-infrared (IR) counterpart is an early B-type supergiant star with numerous emission lines (Filliatre \& Chaty 2004). Interestingly the obscuration toward IGR J16318-4848 obtained in the optical and near-IR wave bands $\left(A_{V} \sim 18\right)$ is almost 2 orders of magnitude smaller than that inferred from the X-rays, which suggests that the extreme obscuration seen in the X-ray is intrinsic only to the X-ray source. However, $A_{V} \sim 18$ is still greater than the interstellar obscuration, indicating the existence of substantial circumstellar material around the supergiant companion.

In order to fully understand the implications of the INTEGRAL discoveries - specifically, whether they imply existence of a separate class of highly obscured X-ray binaries - we must investigate any similarities between the new INTEGRAL sources and previously known sources. As suggested by Revnivtsev et al. (2003) the Xray pulsar GX 301-2 appears similar to the new INTEGRAL highly obscured massive $\mathrm{X}$-ray binaries: it has variable $\mathrm{X}$-ray obscuration of $N_{\mathrm{H}} \simeq 10^{23}-10^{24} \mathrm{~cm}^{-2}$, the compact source is a neutron star, and the optical companion is an early B-type supergiant (or hypergiant; Kaper et al. 1995), like IGR J16318-4848. Recently, Kaplan et al.

\footnotetext{
${ }^{1}$ Department of Astronomy and Astrophysics, University of Toronto, Toronto, ON M5S 3H4, Canada; moon@astro.utoronto.ca.

${ }^{2}$ Pappalardo Fellow, Kavli Institute for Astrophysics and Space Research and Department of Physics, Massachusetts Institute of Technology, Cambridge, MA 02139; dlk@space.mit.edu.

${ }^{3}$ Infrared Processing Analysis Center, California Institute of Technology, MS 220-6, Pasadena, CA 91125; reach@ipac.caltech.edu.

${ }^{4}$ Space Radiation Laboratory, California Institute of Technology, MC 22047, Pasadena, CA 91125; fiona@srl.caltech.edu.

${ }^{5}$ Department of Astronomy and Space Science, Sejong University, Seoul 143-747, Korea; jelee@ sejong.ac.kr.

${ }^{6}$ Canadian Institute for Theoretical Astrophysics, University of Toronto, Toronto, ON M5S 3H8, Canada; pgmartin@ cita.utoronto.ca.
}

(2006, hereafter Paper I), using optical, near-IR, and mid-IR $(\leq 20$ $\mu \mathrm{m})$ spectral energy distributions (SEDs), found that both sources have strong mid-IR excesses that they identify as continuum emission from hot dust. This suggests that they have very similar circumstellar material which may be related to their strong X-ray obscuration. In this Letter, we present the results of Spitzer mid-IR spectroscopic observations of IGR J16318-4848 and GX 301-2, showing that both sources indeed have very similar rich mid-IR properties previously unknown for X-ray binaries.

\section{OBSERVATIONS AND DATA REDUCTION}

We observed IGR J16318-4848 and GX 301-2 with the InfraRed Spectrograph (IRS) aboard Spitzer (Houck et al. 2004) on 2005 September 8 and 2005 July 2, respectively. The duration of the observations was $1.32 \mathrm{hr}$ (IGR J16318-4848) and $0.82 \mathrm{hr}$ (GX 301-2) with the all six IRS modules in the staring mode, obtaining two nods of spectra separated by one-third of the slit length. For data analysis, we used Basic Calibration Data from the standard data reduction pipeline (S13.2.0) and the Spitzer IRS Custom Extraction (SPICE) software (v1.3-beta1). In the lowresolution mode where the slit lengths are large (i.e., $57^{\prime \prime}$ for 5.2$14.5 \mu \mathrm{m} ; 168^{\prime \prime}$ for $14.0-38.0 \mu \mathrm{m}$ ), we subtracted out background emission of one nod using the spectrum of the another nod, and combined them for the final spectrum. In the high-resolution mode, however, the background subtraction was impossible due to the small slit length (i.e., $11.3^{\prime \prime}$ for 9.9-19.6 $\mu \mathrm{m}$; 22.3" for 18.7-37.2 $\mu \mathrm{m})$ and high brightnesses of the sources. Therefore, we just combined the spectra from the two nods for the final high-resolution spectrum without any background subtraction. We used IRSCLEAN (v1.5) to mask and clean rogue pixels, and then interpolated over neighboring normal pixels.

\section{LOW-RESOLUTION SPECTRUM: CONTINUUM AND BROAD FEATURES}

Some emission features, such as ionic forbidden lines, $\mathrm{H}_{2}$ lines, and polycyclic aromatic hydrocarbon (PAH) emission, present in the low-resolution spectra appear extended. Here we consider just 


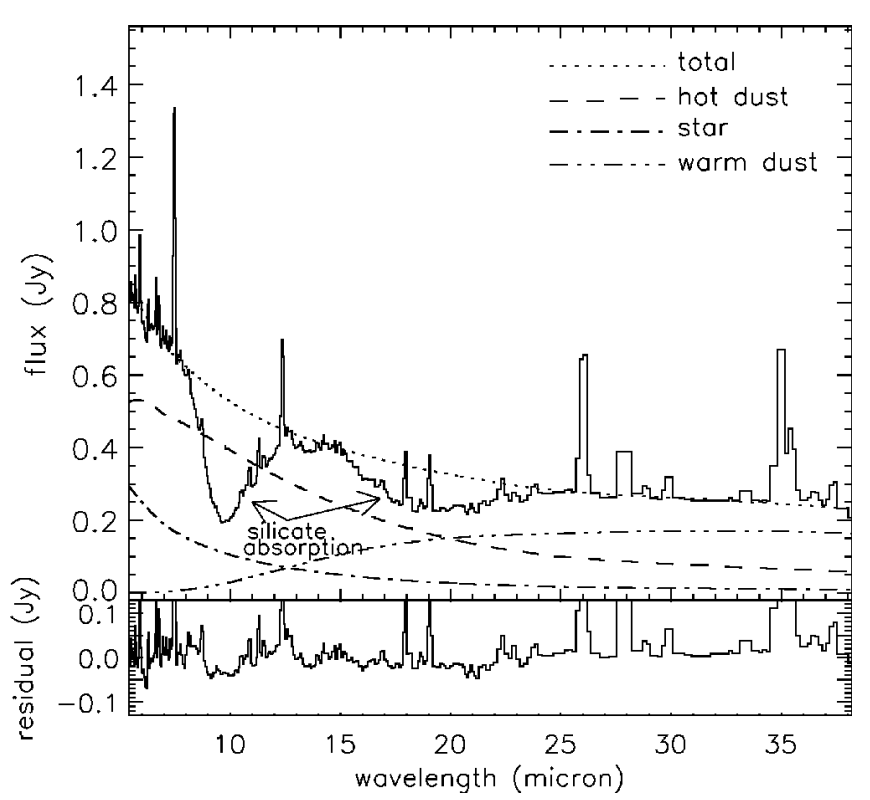

FIG. 1.-Top: Background-subtracted IRS low-resolution spectrum of IGR J16318-4848. The solid line represents the observed spectrum, while the dotted line shows the best-fit spectrum combining the hot-dust (dashed line), the stellar (dot-dashed line), and the warm-dust (double-dot-dashed line) components. The SED fit was conducted excluding the silicate absorption features indicated by the arrows. Bottom: Residuals of the SED fits. Note that for the residual the fluxes of the wavelengths associated with the silicate absorption features were calculated with the best-fit parameters (see text).

the emission from point sources, and defer discussion of the extended emission to a separate paper. Figure 1 presents the background-subtracted low-resolution spectrum of IGR J16318-4848, as well as the best-fit spectra of the continuum emission and the residual of the SED fit (see below). Note that the low-resolution spectrum is extracted for the continuum source (i.e., IGR J16318-4848) based on the standard point source extraction procedure of SPICE. Several features are prominent in Figure 1, including complicated multicomponent continuum emission, broad and strong absorption around 9.7 and $18 \mu \mathrm{m}$, and numerous emission lines. The spectrum of GX 301-2 (Fig. 2) is similar, with strong continuum emission, silicate absorptions feature around 9.7 $\mu \mathrm{m}$, and several emission lines.

As in Figures 1 and 2, the silicate absorption and emission line features make it difficult to carry out any meaningful independent SED fits in the shorter wavelength $(\lambda \leq 8 \mu \mathrm{m})$ range. Thus, instead, we first examined whether the IRS SEDs of the shorter wavelengths are consistent with the results of Paper I using only the line-free regions. According to Paper I, the SEDs of IGR J16318-4848 and GX 301-2 at $\lambda \leq 8 \mu \mathrm{m}$ have contributions from hot (i.e., $T \simeq 1040$ $\mathrm{K}$ for IGR J16318-4848 and $720 \mathrm{~K}$ for GX 301-2) dust emission and stellar emission of a B0 I star with temperature of $26,000 \mathrm{~K}$, while the contribution of free-free emission is negligible. Our IRS SEDs of the line-free regions in Figures 1 and 2 at $\lambda \leq 8 \mu \mathrm{m}$ were fitted very well with those parameters, resulting in $\chi_{\nu}^{2} \leq 1$. We used the extinction $A_{V}=18.5$ (IGR J16318-4848) and 7.1 (GX 301$2)$ as in Paper I. However, in the longer wavelength $(\lambda \geq 22 \mu \mathrm{m})$ range, we were unable to fit the IRS SEDs with the given hot dust and stellar parameters because the best fit resulted in unacceptably large $\chi^{2}$ values. We therefore performed SED fits with three components (i.e., hot-dust, stellar, and warm-dust components) using the entire line-free SEDs in the $\lambda \leq 8 \mu \mathrm{m}$ and $\geq 22 \mu \mathrm{m}$ range. Over the fits the parameters of the warm-dust component were treated as free parameters, while those of the hot-dust and stellar components were fixed to be the values in Paper I. As a result, we obtained warm-

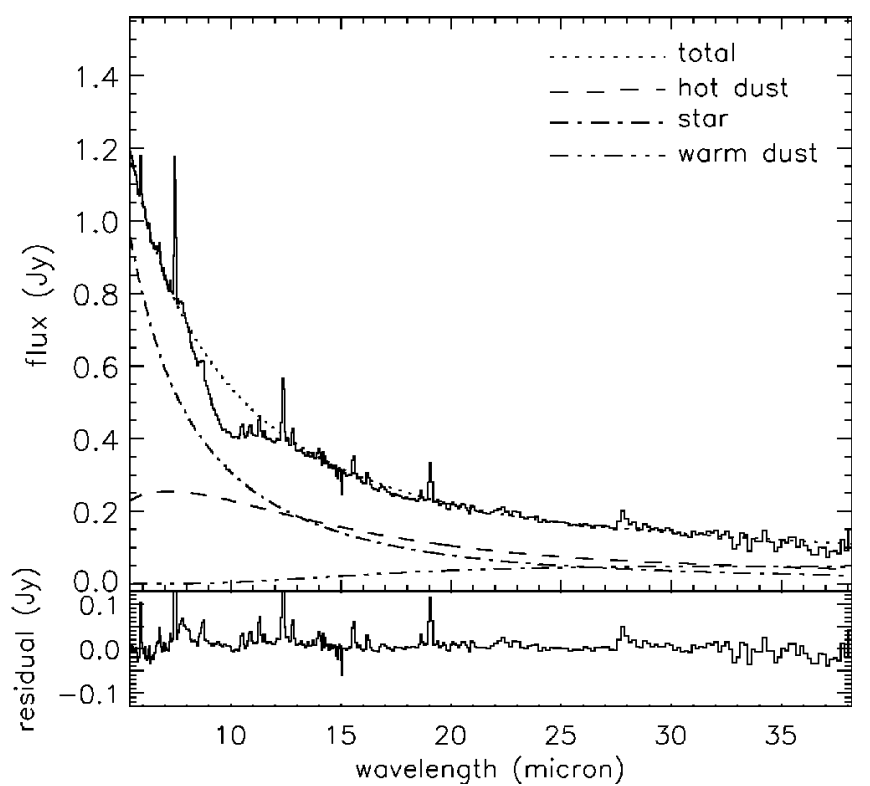

FIG. 2.-Same as Fig. 1, but for GX 301-2. Note the silicate absorption around $9.7 \mu \mathrm{m}$.

dust temperatures of $190 \mathrm{~K}$ (IGR J16318-4848) and $170 \mathrm{~K}$ (GX 301-2) with $\chi_{v}^{2} \simeq 1.2$ (IGR J16318-4848) and 1.5 (GX 301-2). We used the extinction coefficients compiled in Mathis (2000), excluding the $\lambda=8-22 \mu \mathrm{m}$ range to avoid the uncertainties of the coefficients associated with the silicate absorption features. We instead interpolated the extinction coefficients at $\lambda \leq 8$ and $\geq 22 \mu \mathrm{m}$ to calculate the silicate-absorption-free extinction coefficients in the $\lambda=8-22 \mu \mathrm{m}$ range, and used them in the fits. The top panels of Figures 1 and 2 present the results of our best SED fit obtained by excluding the silicate absorption features, where we can confirm that the fit matches nicely the observed SED except the $\lambda=8-22$ $\mu \mathrm{m}$ range. In the bottom panel, on the other hand, we show the residuals of the fit obtained by subtracting the fluxes computed by the best fit from the observed values. For this residual construction, in order to see whether our best SED fit is consistent with the observed silicate absorption features, we combined the best-fit parameters obtained at $\lambda \leq 8$ and $\geq 22 \mu \mathrm{m}$ with the extinction coefficients in the $\lambda=8-22 \mu \mathrm{m}$ range (Mathis 2000), and calculated the silicate-absorption-feature-associated fluxes expected by the best SED fit in the $\lambda=8-22 \mu \mathrm{m}$ range. The residuals in Figures 1 and 2 show that overall our best SED fit agrees with the silicate absorption features reasonably well, although there appears to be some small discrepancies for IGR J16318-4848.

The masses of the dust components were estimated under the optically thin assumption (Hildebrand et al. 1977): $M_{\text {dust }}=$ $F_{\lambda} d^{2} / \kappa_{\lambda} B_{\lambda}\left(T_{\text {dust }}\right)$, where $d$ is the distance to the source, $\kappa_{\lambda}$ is the dust mass absorption coefficient, and $B_{\lambda}(T)$ is the Planck function at temperature $T$. Using the interstellar mass absorption coefficients of Draine (2003) at 8 and $25 \mu \mathrm{m}$, the hot- and warm-dust masses are estimated to be $M_{\mathrm{hot}} \simeq 4.5 \times 10^{-9} d_{5}^{2} M_{\odot}$ and $M_{\mathrm{warm}} \simeq$ $3.1 \times 10^{-7} d_{5}^{2} M_{\odot}$ for IGR J16318-4848, and $M_{\text {hot }} \simeq 5.7 \times$ $10^{-9} d_{5}^{2} M_{\odot}$ and $M_{\text {warm }} \simeq 1.4 \times 10^{-7} d_{5}^{2} M_{\odot}$ for GX 301-2, where $d_{5}$ is the distance to the sources normalized by $5 \mathrm{kpc}$.

The silicate absorption features seen in Figures 1 and 2 have a tight linear correlation with the optical extinction in the diffuse interstellar medium (ISM), although the correlation has been reported to break down in dense environments where the silicate feature does not show a monotonic increase with extinction at $A_{V}$ $\geq 12$ mag (e.g., Chiar et al. 2007). The correlation in the diffuse 


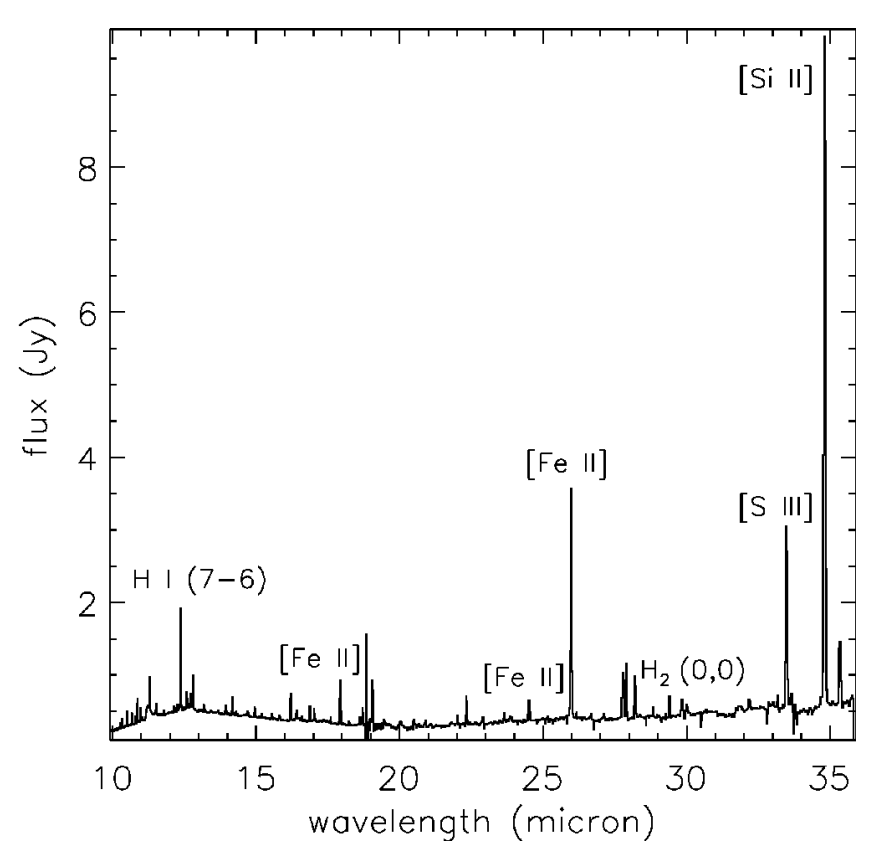

FIG. 3.-IRS high-resolution spectrum of IGR J16318-4848. Note that the background is not subtracted. Some strong lines are marked.

ISM is $A_{V} / \tau_{9.7}=18.5$ (Draine 2003 and references therein; $\tau_{9.7}$ is the optical depth of the silicate absorption at $9.7 \mu \mathrm{m}$ ). If we adopt this relation, the intensity ratios of the observed spectrum to the unabsorbed spectrum in Figures 1 and 2 at $9.7 \mu \mathrm{m}$ correspond to $A_{V} \sim 18.5$ (IGR J16318-4848) and $\sim 6$ (GX 301-2). These are very similar to the values obtained in previous studies for the total optical extinctions of both sources, suggesting that a substantial portion, if not all, of the silicate features seen in Figures 1 and 2 are associated with the optical extinctions of IGR J16318-4848 and GX 301-2.

\section{HIGH-RESOLUTION SPECTRUM: LINE EMISSION}

Figures 3 and 4 present the high-resolution spectra of IGR J16318-4848 and GX 301-2, revealing numerous emission lines. (Note that the background emission is not subtracted.) The majority

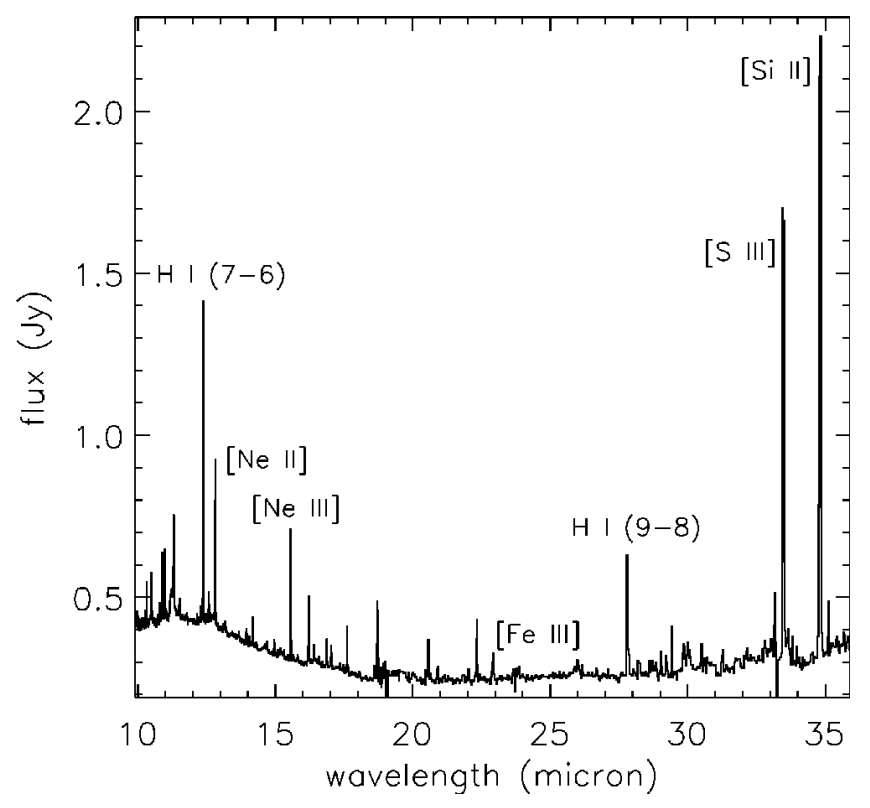

FIG. 4.- Same as Fig. 3, but for GX 301-2.
TABLE 1

OBSERVED INTENSITIES OF H I TRANSITIONS

\begin{tabular}{lrc|lrr}
\hline \hline & IGR & GX & & IGR & \multicolumn{1}{c}{ GX } \\
Transition $(\lambda)^{\mathrm{a}}$ & J16318 & 301-2 & Transition $(\lambda)^{\mathrm{a}}$ & J16318 & $301-2$ \\
\hline $17-9(10.26)$ & 4.39 & 2.44 & $12-8(10.50)$ & 11.57 & 11.40 \\
$16-9(10.80)$ & 5.60 & 2.20 & $9-7(11.31)$ & 25.43 & 13.50 \\
$22-10(11.49)$ & 1.74 & 0.78 & $15-9(11.54)$ & 6.48 & 1.87 \\
$20-10(12.16)$ & 2.84 & 1.35 & $7-6^{\mathrm{b}}(12.37)$ & 72.02 & 42.12 \\
$18-10(13.19)$ & 5.15 & 1.70 & $13-9(14.18)$ & 10.61 & 3.37 \\
$23-11(14.30)$ & 2.00 & 0.93 & $22-11(14.71)$ & 5.02 & 0.87 \\
$16-10(14.96)$ & 5.41 & 1.75 & $20-11(15.82)$ & 2.79 & 1.33 \\
$10-8(16.21)$ & 14.66 & 7.32 & $15-10(16.41)$ & 3.83 & 1.83 \\
$19-11(16.59)$ & 2.15 & 0.40 & $12-9(16.88)$ & 7.35 & 3.17 \\
$14-10(18.62)$ & 4.04 & 1.38 & $16-11(20.92)$ & 2.69 & 1.14 \\
$9-8(27.80)$ & 18.71 & 8.86 & $\ldots$ & $\ldots$ & $\ldots$ \\
\hline
\end{tabular}

Note. - Intensities are in units of $10^{-14} \mathrm{ergs} \mathrm{s}^{-1} \mathrm{~cm}^{-2}$.

${ }^{\mathrm{a}}$ Wavelengths are in units of $\mu \mathrm{m}$.

b The H I 11-8 transitions may be blended.

of them are $\mathrm{H}$ I lines, while the rest consist of ionic forbidden lines, pure rotational $\mathrm{H}_{2}$ lines, and the $11.3 \mu \mathrm{m}$ PAH emission feature. Table 1 lists the intensities of some of the bright $\mathrm{H}$ I lines identified in both sources with high ( $>3$ ) signal-to-noise ratio. The line intensities and the signal-to-noise ratio were estimated with Gaussian profile fits. The intensities of the $\mathrm{H}$ I lines are consistent with gas at $T \simeq 10^{4} \mathrm{~K}$ and with $N_{\mathrm{e}} \simeq 10^{4} \mathrm{~cm}^{-3}$ in the Menzel case B state, following Filliatre \& Chaty (2004), although the intensity ratios are not very sensitive to those parameters (Hummer \& Storey 1987).

Table 2 lists the parameters of forbidden ionic lines and $\mathrm{H}_{2}$ lines. For the forbidden lines, [Ne II], [Ne III], [S III], and [Si II] were detected in both sources, while [Ni II] and [Fe II] were only in IGR J16318-4848; [Fe III] is only in GX 301-2. The $\mathrm{H}_{2}$ lines were detected in both sources. The intensity ratio of the [S III] lines of 18.7 and $33.5 \mu \mathrm{m}$ is diagnostic of the number density in the temperature range of 5000-20,000 K. The extinction-corrected [S III] line surface brightness ratio of $18.7 \mu \mathrm{m} / 33.5 \mu \mathrm{m}$ is 1.21 for IGR J16318-4848 and 1.68 for GX 301-2: according to the calculation of Houck et al. (1984) and Alexander et al. (1999), the ratios correspond to the electron number density $N_{e} \sim 1000 \mathrm{~cm}^{-3}$ for both sources. On the other hand, the intensity ratio of the [Ne II] line $(12.8 \mu \mathrm{m})$ and the $[\mathrm{Ne} \mathrm{III}]$ line $(15.6 \mu \mathrm{m})$ is sensitive to the hardness of the radiation, or temperature of the environment. If we use the calculation of Kunze et al. (1996) the extinction-corrected intensity ratio of $[\mathrm{Ne} \mathrm{II}] /[\mathrm{Ne} \mathrm{III}]$ is consistent with $\sim 4 \times 10^{4} \mathrm{~K}$ for IGR $\mathrm{J} 16318-4848$ and $\sim 4.5 \times 10^{4} \mathrm{~K}$ for GX 301-2. The three $\mathrm{H}_{2}$ lines detected in the both sources are pure rotational transition lines of $\mathrm{H}_{2}(0,0) \mathrm{S}(0,1,2)$, which are usually excited by collisions. Assuming that the bottom two lines follow the distribution of the local

TABLE 2

OBSERVED INTENSITIES OF OTHER LINES

\begin{tabular}{|c|c|c|c|c|c|}
\hline Line $(\lambda)^{\mathrm{a}}$ & $\begin{array}{c}\text { IGR } \\
\text { J16318 }\end{array}$ & $\begin{array}{c}\text { GX } \\
301-2\end{array}$ & Line $(\lambda)^{\mathrm{a}}$ & $\begin{array}{c}\text { IGR } \\
\text { J16318 }\end{array}$ & $\begin{array}{c}\text { GX } \\
301-2\end{array}$ \\
\hline [Ni II] (10.68) & 10.25 & $\ldots$ & [Ni II] (12.73) & 13.33 & . \\
\hline [Ni II] (18.24) & 1.69 & & & $\ldots$ & \\
\hline$[\mathrm{Ne}$ II $](12.81)$ & 21.44 & 23.41 & {$[\mathrm{Ne}$ III $](15.56)$} & 2.60 & 15.67 \\
\hline [Fe II] (17.94) & 22.54 & $\ldots$ & {$[\mathrm{Fe}$ II $](24.52)$} & 7.33 & $\ldots$ \\
\hline [Fe II] (25.99) & 70.93 & & {$[\mathrm{Fe}$ II] $(35.35)$} & 15.46 & \\
\hline [Fe III $](22.93)$ & & 1.99 & & & \\
\hline [S III] (18.71) & 7.73 & 8.02 & [S III] (33.48) & 40.14 & 25.01 \\
\hline [Si II] (34.82) & 177.20 & 33.33 & & $\ldots$ & $\ldots$ \\
\hline $\mathrm{H}_{2}(0,0) S(2)$ & & & $\mathrm{H}_{2}(0,0) S(1)$ & & \\
\hline (12.28) & 2.88 & 1.44 & (17.03) & 4.66 & 2.05 \\
\hline $\begin{array}{l}\mathrm{H}_{2}(0,0) S(0) \\
\quad(28,22)\end{array}$ & & & & & \\
\hline$(28.22)$ & 10.08 & 4.24 & $\ldots$ & $\ldots$ & $\ldots$ \\
\hline
\end{tabular}

Note. - Intensities are in units of $10^{-14} \mathrm{ergs} \mathrm{s}^{-1} \mathrm{~cm}^{-2}$.

${ }^{\mathrm{a}}$ Wavelengths are in units of $\mu \mathrm{m}$. 
thermodynamic equilibrium and are optically thin, the surface brightness ratios are consistent with the excitation temperature $T_{\mathrm{ex}} \sim 1000$ $\mathrm{K}$. The estimated $\mathrm{H}_{2}$ column densities are $\sim 1 \times 10^{19} \mathrm{~cm}^{-2}$ (IGR $\mathrm{J} 16318-4848$ ) and $5 \times 10^{18} \mathrm{~cm}^{-2}$ (GX 301-2). This implies that the optical extinction associated with the warm molecular gas is very small, $A_{V} \ll 1$, based on the relation $N_{\mathrm{H}}=1.87 \times 10^{21} A_{V}$ (Draine 2003).

\section{DISCUSSION AND CONCLUSION}

Our Spitzer spectroscopic observations of IGR J16318-4848 and GX 301-2 have revealed for the first time the rich mid-IR environment of highly obscured X-ray binaries. This includes two dust components with prominent silicate absorption, numerous $\mathrm{H}$ I recombination lines, many forbidden ionic lines, and pure rotational $\mathrm{H}_{2}$ lines. Based on the observed spectra, we infer the following components for IGR J16318-4848 and GX 301-2: (1) hot $(T>700 \mathrm{~K})$ and warm $(T \sim 180 \mathrm{~K})$ circumstellar dust; $(2)$ ionized stellar winds responsible for the $\mathrm{H}$ I lines; (3) extended low-density ionized regions for the forbidden lines; and (4) photodissociated regions associated with the $\mathrm{PAH}, \mathrm{H}_{2}$, and possibly [Si II] line emission. For the forbidden lines, all the detected lines have relatively low ionization potential like in starburst galaxies, where the radiation is relatively soft (compared with active galactic nuclei, for instance). This may indicate that the illumination of hard X-rays from the central compact X-ray source is not primarily responsible for the forbidden line emission. However, the inferred temperature for the radiation exciting the $[\mathrm{Ne}$ II] and [Ne III] lines is hotter than the stellar photospheres, so there can be some contribution from the compact object. Considering that [Ni II] and [Fe II] were detected only in IGR J16318-4848 while [Fe III] was only in GX 301-2, the radiation field of IGR J16318-4848 may be softer than GX 301-2, as demonstrated by the small temperature difference between the two sources (see $\S 4$ ).

Perhaps the most natural explanation for the origin of the hotdust component relies on dust formation in the dense outflows from the early-type companions of IGR J16318-4848 and GX $301-2$, as is the case in most $\operatorname{sgB}[\mathrm{e}]$ stars (i.e., B-type supergiants with forbidden emission lines). However, the origin of the warm circumstellar dust component is very uncertain, and B[e] stars seldom show evidence for this type of warm dust. If both the hotand warm-dust components have spherical shell geometries around the central star, then the associated optical extinctions are $A_{V} \simeq 4$ $\times 10^{-4} Q_{\text {abs }}\left(M_{d} / 10^{-6} M_{\odot}\right)\left(T_{d} / 100 \mathrm{~K}\right)^{4}\left(L_{\mathrm{UV}} / 10^{39} \mathrm{ergs} \mathrm{s}^{-1}\right)$, where $M_{d}$ and $T_{d}$ are the mass and temperature of the dust components, $Q_{\text {abs }}<1$ is the dust absorption coefficient, and $L_{\mathrm{UV}}$ is the ultraviolet luminosity of the central star. This gives the optical extinctions $A_{V} \ll 1$ for both the hot- and warm-dust components of IGR J16318-4848 and GX 301-2. (Here we use $1 \times 10^{39} \mathrm{ergs} \mathrm{s}^{-1}$ for $L_{\mathrm{UV}}$ for both sources.) Therefore, under the assumption of spherical shell geometry, both the hot and warm dust components of IGR J16318-4848 and GX 301-2 contribute very little to the total optical extinction, suggesting that the hot- and warm-dust components are not strongly associated with the silicate absorption features. This also applies to the dust associated with the warm extended $\mathrm{H}_{2}$ gas since the optical extinction from this component is tiny (i.e., $A_{V} \ll 1$; see $\S 4$ ). What is then the origin of the silicate absorption features? If it is due to the foreground ISM, we would expect to see the $\mathrm{CO}_{2}$ ice feature around $15 \mu \mathrm{m}$, especially for IGR J16318 -4848, based on the intensity ratio between the silicate absorption feature and ice feature found in the ISM (Knez et al. 2005). The absence of the ice feature in our spectra supports the interpretation that the silicate absorption features are probably not associated with the foreground ISM. One possibility may be the existence of an undisclosed colder (e.g., $\ll 100 \mathrm{~K}$ ) circumstellar dust component which is responsible for the silicate absorption features and most of the optical extinction. We need further longer wavelength observations to confirm this possibility. Considering that the $9.7 \mu \mathrm{m}$ silicate absorption feature represents the oxygenrich material, the existence of the $9.7 \mu \mathrm{m}$ silicate absorption feature may indicate that the origin of the potential colder dust component is related to the nucleosynthesis of the progenitors of IGR J16318-4848 and GX 301-2 (or their companions).

The optical/near-IR companion of IGR J16318-4848 is a sgB[e] star. Such stars are known to have hot $(T \sim 1000 \mathrm{~K})$ circumstellar dust, and probably are evolving into luminous blue variables (LBVs) or Wolf-Rayet stars. Our mid-IR spectra of IGR J16318-4848 and GX 301-2 are very similar to that of the LBV P Cygni, which shows many H I lines and forbidden ionic lines (Lamers et al. 1996b). The difference is that while the mid-IR continuum of P Cygni is due to free-free emission in stellar winds, the main mid-IR emission of IGR J16318-4848 and GX 301-2 is from multiple dust continua. (The SEDs of both sources observed here are inconsistent with freefree emission, as mentioned in Paper I.) However, we note that some LBV stars have also been observed to have thermal mid-IR dust emission (Lamers et al. 1996a). Based on the fact that B[e] stars seldom show mid-IR forbidden line emission, the B-type supergiant (or hypergiant) companions of IGR J16318-4848 and GX 301-2 may be on the evolutionary track to LBVs, implying that the extremely high obscuration seen in some massive X-ray binaries may be a phenomenon associated with the evolutionary phase. This scenario is also consistent with the fact that the highly obscured massive X-ray binary Cygnus X-3 has a Wolf-Rayet star companion, together with the circumstellar dust emission of $T \sim 250 \mathrm{~K}$ (Koch-Miramond et al. 2002).

This work is based on observations made with the Spitzer Space Telescope, which is operated by the Jet Propulsion Laboratory, California Institute of Technology, under a contract with NASA. D.-S. M. thanks Elise Furlan for her help in IRS data analysis, and Marten van Kerkwijk for comments. This research was partly supported by a Discovery Grant (327277) of the Natural Science and Engineering Research Council of Canada to D.-S. M.

\section{REFERENCES}

Alexander, T., et al. 1999, ApJ, 512, 204

Chiar, J. E., et al. 2007, ApJ, 666, L73

Courvoisier, T. J.-L., et al. 2003, IAU Circ., 8063, 3

Draine, B. T. 2003, ARA\&A, 41, 241

Filliatre, P., \& Chaty, S. 2004, ApJ, 616, 469

Hildebrand, R. H., et al. 1977, ApJ, 216, 698

Houck, J. R., Shure, M. A., Gull, G. E., \& Herter, T. 1984, ApJ, 287, L11

Houck, J. R., et al. 2004, ApJS, 154, 18

Hummer, D. G., \& Storey, P. J. 1987, MNRAS, 224, 801

Kaper, L., et al. 1995, A\&A, 300, 446

Kaplan, D. L., Moon, D.-S., \& Reach, W. T. 2006, ApJ, 649, L107 (Paper I)
Knez, C., et al. 2005, ApJ, 635, L145

Koch-Miramond, L., et al. 2002, A\&A, 396, 877

Kunze, D., et al. 1996, A\&A, 315, L101

Lamers, H. J. G. L. M., et al. 1996a, A\&A, 315, L225 1996b, A\&A, 315, L229

Mathis, J. S. 2000, in Allen's Astrophysical Quantities, ed. A. N. Cox (London: Athlone Press), 527

Revnivtsev, M. G., Sazonov, S. Yu., Gilfanov, M. R., \& Sunyaev, R. A. 2003, Astron. Lett., 29, 587

Walter, R., et al. 2003, A\&A, 411, L427

Winkler, C., et al. 2003, A\&A, 411, L349 